\title{
A cross-sectional Study of Metamemory in Obese South Indian Females
}

\author{
G.Geethika ${ }^{1}$, A.Sangeetha ${ }^{2}$, Kumaresan $M^{3}$, Hemachandrika $C^{4}$, Karthika $\mathbf{M}^{5}$ \\ ${ }^{1}$ II MBBS Student, ${ }^{2}$ Assistant Professor, Department of Physiology, ${ }^{3}$ Assistant Professor, Department of Anatomy, \\ , 'Professor \& Head, Department of Physiology, Saveetha medical College and hospital, Chennai, Tamilnadu, \\ India, ${ }^{5}$ Assistant Professor, Department of Physiology, Dhanalakshmi Srinivasan Medical College and Hospital, \\ Peramablur, Tamilnadu, India
}

\begin{abstract}
Introduction: Metamemory is the introspective knowledge of one's brain memory capability and process involved in memory self-monitoring. This self-awareness of memory has important implications for how people learn and use memories for everyday activities. Aim: The aim is to assess metamemory in female obese individuals. Materials and Method: A study was conducted among 165 female overweight and obese category at random south Indian population. The participants were asked to complete a self-reported questionnaire on metamemory. The Multifactorial Memory Questionnaire (MMQ) was developed to assess separate dimensions of memory ratings that are applicable to clinical assessment and intervention, includes scales of Contentment (i.e., affect regarding one's memory), Ability (i.e., self-appraisal of one's memory capabilities), and Strategy (i.e., reported frequency of memory strategy used). Result: Association between corresponding subscale scores and blood glucose which indicates a weak correlation between blood glucose, satisfaction $(r=-.008)$ and strategy $(r=-.07)$. In addition, the MMQ subscales and the total score showed good internal consistency ( $\alpha$ s $=0.82-0.85$ ). Conclusion: Obese and overweight female individuals had poor metamemory scores. The study would help the obese individuals to identify any early stage of cognitive impairment and create an awareness to delay or prevent any further metacognitive dysfunction.
\end{abstract}

Keywords: Metamemory, obese, dementia, Hippocampus

\section{Introduction}

Metamemory is defined individual's knowledge about functioning of memory in general than individual knowledge of their own memory. In this context metamemory is the introspective knowledge of one's brain memory capability and process involved in memory self-monitoring ${ }^{[1]}$. This self-awareness of memory has important implications for how people learn and use memories. For example, when a person asserts that he or she is good at remembering faces, but poor at remembering names, that person is making a statement concerning metamemory knowledge. Metamemory awareness refers to our feelings or experiences of our own memory. For example, if a person feels certain that he or she will remember later something just learned now, that person is having a metamemory experience [2-5]. Metamemory is a subarea of Meta cognitions. Accordingly refers to people's self-monitoring and selfcontrol of their own memory process and strategies that can aid memory. Due to a sedentary lifestyle, more and more people are becoming obese nowadays. In addition to health-related problems, obesity can also impair cognition and motor performance ${ }^{(6)}$.

Overweight and obesity are usually related to poorer cognition across lifespan ${ }^{(7-9)}$. It is increasingly evident that obesity negatively impacts human health and the prevalence of obesity is increasing world-wide ${ }^{[10]}$. Both overall obesity (body mass index $(\mathrm{BMI})>30$ $\mathrm{kg} / \mathrm{m}^{2}$ ) and fat distribution (waist-hip-ratio (WHR) $>1.0$ in men and $>0.85$ in women indicative of abdominal 
fat accumulation) have been linked to cardiometabolic diseases and death in observational studies (11-14). Compared to BMI, central adiposity has a stronger association with the risk of developing cognitive impairment and dementia in women ${ }^{(15)}$. Therefore it is important to evaluate the state of metamemory in obese female individuals. This will not allow them to bring back their memory to full extent but it will at least be useful for them to look for alternatives to make sure they don't forget events happening every day. So, the state of metamemory in overweight or obese person will surely result in better understanding of the cognitive condition by the physicians. Therefore, the purpose of this study is to investigate and understand the relation between the decrease or increase in metamemory in obese female individuals.

\section{Materials And Method}

\section{Ethical Consideration:}

The study proposal was approved by the board of the Saveetha medical college and hospitals (IRB No. SMC/IEC/2020/03/028). The purpose and objective of the study was clearly explained to the participants through an information sheet. It was emphasized that their participation was optional and the confidentiality of data was assured. The participants were requested to sign a consent form attached with the questionnaire, to ensure their willingness to participate in the study.

\section{Study setting and design:}

This was a cross-sectional, descriptive correlational study. This standard questionnaire was done by 165 female obese individuals who volunteered to participate in this study. A convenience sample of participants from 19 to 55 years old female individuals was recruited from Medicine OP, Saveetha Hospitals. The questionnaire was administered through face-to-face contact by the investigator with potential participants. Potential participants who expressed interest in the study were screened for eligibility based on the inclusion/exclusion criteria. Inclusion criteria were as follows: ages from 19 to 55 ; BMI ranges between 30 to 40 ; ability to read, speak, and understand english. Participants with comorbidities like diabetes mellitus, hypertension and hyperlipidemia were excluded. Individuals with cognitive impairment (Alzheimer's disease) were also excluded. After the inclusion/exclusion criteria were applied, 120 participants have received paper copies of the study's survey instrument. The questionnaire included about age, height and weight, hence BMI calculated BMI = Weight/Height in meter ${ }^{2}$. The participant's BMI ranges are between 30 and 40. Individuals falling under obese I \& II category were included.

\section{Procedure:}

\section{Perceived memory:}

The multifactorial memory questionnaire is a standard metamemory questionnaire (MMQ) which helps to assess a Metamemory of a person. It consists of three scales measuring separate aspects of metamemory. Items are rated on a 5 -point Likert scale $(0=$ strongly agree, 1 = agree, $2=$ undecided, $3=$ disagree, $4=$ strongly disagree) based on the test's takers experiences. The three MMQ scales and their respective metamemory domains include: MMQ-Satisfaction (formerly called MMQContentment). This scale measures satisfaction, concern, and overall appraisal of one's own memory. Each of 18 statements is rated based on degree of agreement. The score range is 0 to 72 , with higher scores indicating a higher degree of satisfaction. MMQ-Ability. This scale measures self- perception of everyday memory ability. Respondents rate how often they experienced each of 20 common memory mistakes over the previous two weeks. The score range is 0 to 80 , with higher scores indicating better self-reported memory ability. MMQ-Strategy. This scale measures the use of practical memory strategies and aids in day-to-day life. Respondents rate how often they used each of 19 memory strategies over the previous two weeks. The score range is 0 to 76 , with higher scores indicating greater use of memory strategies. Based on questionnaire data total score ranges are measured. Using a method formula; Prorated Score $=$ Number of possible items X (Obtained score/ Number of completed items). 


\section{Data Analysis}

Statistical analysis was done using SPSS Version 25.0. Descriptive variables were reported (Mean with standard deviation, Percentage) for all demographic variables. Pearson's correlation analysis was used to assess correlations between BMI and the survey scores (Satisfaction, ability and strategy) and Cronbach's alpha was calculated to measure internal consistency among the individual scores ${ }^{(16)}$. The significance level was set at 0.05 .

\section{Results}

Among the 165 participants, the mean and SD for age, height \& weight were calculated (Table 1). Mean and SD for blood glucose levels and MMQ Subscale scores (Satisfaction, ability and strategy) are given in Table 2. Age and BMI were correlated with MMQ subcomponents (Table 3). The internal consistency of subscale scores are measured by cronbach's alpha to check the reliability. There was a weak negative relationship between age and MMQ subscales (Satisfaction, ability and strategy). This relationship suggests that in obese female individuals, increasing age is associated with decreased satisfaction, metamemory. Based on the MMQ subcomponent scores the study participants were found to have more worries about their memory (MMQcontentment), report significantly more instances of forgetfulness (MMQ-ability), and use less memory aid strategies in their day-to-day activities (MMQ-strategy) (Table $2 \& 3$ ). BMI had a weak negative correlation with MMQ-contentment ( $r=-.008)$ or MMQ-ability ( $r=$ $.03)$ or MMQ-strategy $(r=.07)$. In our evaluation with a sample of 165 middle-aged and older obese female individuals analyses using Cronbach's alpha indicated good internal consistency for the Satisfaction $(\alpha=.85)$, Ability $(\alpha=.84)$, and Strategy $(\alpha=.82)$ scales (Table 3 ).

Table 1: Demographic characteristics of the participants

\begin{tabular}{|c|c|c|c|}
\hline & Mean & SD & Range \\
\hline Age (Yrs) & 44.62 & 14.6 & $19-70$ \\
\hline Height (Cms) & 155.88 & 4.1 & $142-165$ \\
\hline Weight (Kgs) & 83.12 & 9.7 & $55-109$ \\
\hline
\end{tabular}

Table 2: Summary statistics for blood glucose levels and MMQ raw scores:

\begin{tabular}{|c|c|c|c|}
\hline Scale & Mean & SD & SEM \\
\hline BMI & 34.51 & 3.6 & 0.47 \\
\hline Satisfaction & 49.87 & 10.2 & 1.3 \\
\hline Ability & 48.98 & 10.7 & 1.3 \\
\hline Strategy & 49.44 & 10.3 & 1.4 \\
\hline
\end{tabular}


Table 3: Correlations between demographic characteristics and cognitive variables \& internal consistency of MMQ subscales

\begin{tabular}{|c|c|c|c|}
\hline Scale & Age & BMI & Cronbach's $\alpha$ \\
\hline Satisfaction & $r=-.14$ & $r=-.008$ & .85 \\
\hline Ability & $r=-.17$ & $r=-.03$ & .84 \\
\hline Strategy & $r=-.13$ & $r=-.07$ & .82 \\
\hline
\end{tabular}

\section{Discussion}

In the present study, Obesity in female obese individual adults showed a negative correlation on all the metacognitive components. When the BMI values were correlated with metamemory components it showed a weak negative correlation. Simply, the study has indicated that female with overweight and obese report more worries about their memory, more forgetful-ness, and more use of strategies to ameliorate memory difficulties. Based on BMI data, individuals who are overweight or obese, fall in the lowest quartile of global cognition, verbal fluency, delayed recall, immediate logical memory, and intelligence ${ }^{(17)}$. Other than BMI, other adiposity measures are also related to cognitive performance and brain changes. Visceral adiposity is inversely correlated with verbal memory and attention. High visceral adiposity is associated with smaller hippocampus and larger ventricular volume (18). There is also a negative correlation between waistto-hip ratio and hippocampal volume and a positive correlation between waist-to-hip ratio and white matter hyperintensities ${ }^{(19)}$. Statistical parametric mapping has revealed a significant negative correlation between BMI and metabolic activity in prefrontal cortex (Brodmann areas $8,9,10,11,44)$ and cingulate gyrus (Brodmann area 32) but not in other regions ${ }^{(20-22)}$. These results further indicate the urgency of creating awareness on obesity in the society. A host of previous literature has suggested that exercise can improve both obesity-related cognitive and motor declines. As more and more people develop obesity in young age, introducing exercise intervention early would result in the greatest benefits towards good health ${ }^{(6)}$.

\section{Conclusion}

Obesity has become a worrying health and social issue. The current study also has shown that obese and overweight individuals had poor metamemory scores. Obesity affects cognition mainly through altering the brain structures and functions and motor performance. The study would help the obese individuals to identify any early stage of cognitive impairment and create an awareness to delay or prevent any further metacognitive dysfunction. Regular physical activity and exercise benefits both cognition and motor behaviours.

\section{Limitations:}

The small sample size from a single area of the country also limits generalizability. The current study had taken individuals who were obese for past 2 years. BMI measurements were independent of the quantity of total body fat and a number of potential confounders, including age, puberty stage and household income. The study did not differentiate metamemory values between overweight and obesity. Physical activity was not measured. Future research is needed to investigate relationships between these metacognition variables, objective neuropsychological tests, and functional MRI imaging.

Conflict of Interest: No conflict of interest

Funding Sources: Self-funding 


\section{References}

1. Pannu, J. K.; Kaszniak, A. W Metamemory Experiments in Neurological Populations: A Review". Neuropsychology Review. 2005;15 (3): 105-130.

2. Nelson, T.O. Metamemory: A theoretical framework and new findings" (PDF). The Psychology of Learning and Motivation. 26. Academic Press. 1990; pp. 125-173.

3. Fastame, Maria Chiara \& Hitchcott, Paul \& Penna, et al. Does institutionalization influence perceived metamemory, psychological well-being, and working-memory efficiency in Italian elders? A preliminary study. Journal of Clinical Gerontology and Geriatrics. 2016; 7(1); 6-11,

4. Cavanaugh, J.C The Effect of Age on Metamemory for Working Memory New Zealand Journal of Psychology 1982; 28(1):23 .

5. Stevens FC, Kaplan CD et al. How ageing and social factors affect memory. Ageing. 1999; 28(4):37984.

6. Wang C, Chan JS, Ren L, Yan JH. Obesity Reduces Cognitive and Motor Functions across the Lifespan. Neural Plast. 2016;2016:2473081. doi:10.1155/2016/2473081

7. Prickett C., Brennan L., Stolwyk R. Examining the relationship between obesity and cognitive function: a systematic literature review. Obesity Research and Clinical Practice. 2015;9(2):93-113.

8. Gunstad J., Lhotsky A., Wendell C. R., et al Longitudinal examination of obesity and cognitive function: results from the baltimorelongitudinalstudy of aging. Neuroepidemiology. 2010;34(4):222229.

9. Sabia S., Kivimaki M., Shipley M. J., et al Body mass index over the adult life course and cognition in late midlife: the Whitehall II Cohort study. American Journal of Clinical Nutrition. 2009;89(2):601-607.

10. Afshin A, Forouzanfar MH, Reitsma MB, et al. Health Effects of Overweight and Obesity in 195 Countries over 25 Years. N Engl J Med. 2017;377: 13-27.

11. Seidell JC, Oosterlee A, Thijssen MA, Burema J, et al. Assessment of intra-abdominal and subcutaneous abdominal fat: relation between anthropometry and computed tomography. Am J Clin Nutr. 1987;45: $7-13$.

12. Prospective Studies Collaboration. Body-mass index and cause-specific mortality in 900000 adults: collaborative analyses of 57 prospective studies. Lancet. 2009;373: 1083-1096.

13. Taylor AE, Ebrahim S, Ben-Shlomo Y, et al. Comparison of the associations of body mass index and measures of central adiposity and fat mass with coronary heart disease, diabetes, and all-cause mortality: a study using data from 4 UK cohorts. Am J Clin Nutr. 2010;91: 547-556.

14. WHO. Obesity: Preventing and Managing the Global Epidemic. Report of a WHO consultation. Geneva: World Health Organization; 2000.

15. Kerwin DR, Gaussoin SA, Chlebowski RT, et al Interaction between body mass index and central adiposity and risk of incident cognitive impairment and dementia: results from the Women's Health Initiative Memory Study. Women's Health Initiative Memory Study. J Am Geriatr Soc. 2011 Jan; 59(1):107-12.

16. Wessa P. (2017), Cronbach alpha (v1.0.5) in Free Statistics Software (v1.2.1), Office for Research Development and Education, URL https://www. wessa.net/rwasp_cronbach.wasp/

17. Benito-Leon J, Mitchell AJ et al Obesity and impaired cognitive functioning in the elderly: a population-based cross-sectional study Eur J Neurol. 2013 Jun; 20(6):899-906,

18. Isaac V, Sim S, Zheng H, Zagorodnov V, Tai ES, Chee $M$ Adverse Associations between Visceral Adiposity, Brain Structure, and Cognitive Performance in Healthy Elderly. Front Aging Neurosci. 2011; 3(2):12.

19. Jagust W, Harvey D, Mungas D, Haan M Central obesity and the aging brain. Arch Neurol. 2005 Oct; 62(10):1545-8.

20. Nora D. Volkow, Gene-Jack Wang, Frank Telang Inverse Association Between BMI and Prefrontal Metabolic Activity in Healthy Adults; Obesity 2009; 17 (1); 60 - 65.

21. Volkow ND, Gur RC, Wang GJ et al. Association between decline in brain dopamine activity with age and cognitive and motor impairment in healthy 
International Journal of Physiology, July-September 2021, Vol. 9, No. 3

individuals. Am J Psychiatry 1998;155:344-349.

22. Mozley LH, Gur RC, Mozley PD, Gur RE. Striatal dopamine transporters and cognitive functioning in healthy men and women. Am J Psychiatry 2001;158:1492-1499 